\title{
Formulation of Sustained Release Floating Microspheres of Furosemide from Ethylcellulose and Hydroxypropyl Methylcellulose Polymer Blends
}

\author{
Mulugeta Fentie*, Anteneh Belete and Tsige Gebre-Mariam
}

Department of Pharmaceutics and Social Pharmacy, School of Pharmacy, Addis Ababa University, Addis Ababa, Ethiopia

\begin{abstract}
Furosemide is a potent and commonly used loop diuretic. It is absorbed largely in the stomach and upper small intestine. This narrow absorption window is responsible for its low bioavailability of about $50 \%$, and variable and erratic absorption. The objective of the present investigation was to formulate and evaluate floating microspheres of furosemide for prolonged buoyancy with sustained delivery of the drug into the gastric content. Furosemide loaded microspheres were prepared by the solvent evaporation method. The drug entrapment efficiency was high for all of the formulations ranging from 86.2 to $98.4 \%$. The yield of microspheres production was good partticularly at increased EC/HPMC ratio and lower temperatures. Drug amount and EC/HPMC ratio showed highly significant effects $(p<0.0001)$ on cumulative drug release and buoyancy of microspheres. Floating microspheres that effectively sustain the drug release more than $12 \mathrm{~h}$ and exhibit buoyancy of greater than $77 \%$ in $12 \mathrm{~h}$ were developed. Finally the study confirmed that various furosemide loaded EC/HPMC microspheres formulations could be developed that effectively sustain the drug release for a desired period by varing the ratio of EC and HPMC, and drug amount.
\end{abstract}

Keywords: Floating microspheres; Ethylcellulose; HPMC; Solvent evaporation method; Sustained release

\section{Introduction}

Conventional drug delivery systems achieve as well as maintain the drug concentration within the therapeutically effective range needed for treatment only when taken several times a day [1]. In order to avoid the unnecessarily frequent administration, higher cost of therapy and other undesired features of conventional dosage forms, controlled release preparations have been designed [2]. However, these systems have been of limited success in the case of drugs with a poor absorption window throughout the GIT. This has led to the development of gastro retentive dosage forms. Various approaches have been pursued over the last three decades to increase the retention of oral dosage forms in the stomach. The most common gastro retentive approaches used to increase the gastric residence time of pharmaceutical dosage forms include floating systems, swelling systems, bio/mucoadhesive systems and high density systems. Floating dosage forms are the more reliable and commonly used gastro retentive dosage forms. Floating dosage forms can be classified as single-unit and multiple-unit formulations. Single-unit floating formulations are associated with problems such as sticking to the stomach wall, which may have a potential danger of producing irritation, and unreliable and irreproducible residence time in the stomach owing to their fortuitous ('all-or-nothing') release process. On the other hand, multiple-unit floating dosage forms appear to be better suited since they avoid risk of local irritation and 'all-ornothing' release. This reduced risk of 'all-or-nothing' effect reduces the intersubject variability in absorption and lower the probability of dosedumping [3].

Furosemide is absorbed mostly in the stomach and upper small intestine, possibly due to its weak acidic property, pKa 3.8 [4]. This narrow absorption window is responsible for its low bioavailability of about 50\%, and variable and erratic absorption [5]. Other reports indicate a poorer and highly variable oral bioavailability of $37-51 \%$ [4] or $10-100 \%$ [6]. Administration of furosemide as an intravenous infusion has been shown to improve its diuretic and natriuretic activities in comparison to a bolus injection [7]. The narrow absorption window of furosemide in the upper part of the GIT, together with its improved effect upon continuous drug input, provides a rationale for developing a gastroretentive dosage form for this drug. Such a dosage form would be retained for prolonged period of time in the stomach and release the drug in a sustained manner, thus providing the drug continuously to its absorption site in a controlled manner, extending the absorption phase and increasing the duration of the drug effect [5].

Furosemide, like other loop diuretics, acts by inhibiting NKCC2, the luminal $\mathrm{Na}-\mathrm{K}-2 \mathrm{Cl}$ symporter in the thick ascending limb of the loop of Henle. The action on the distal tubules is independent of any inhibitory effect on carbonic anhydrase or aldosterone; it also abolishes the corticomedullary osmotic gradient and blocks negative, as well as positive, free water clearance.

The objective of the present investigation was to formulate and evaluate floating microspheres of furosemide for prolonged buoyancy with sustained delivery of the drug into the gastric content.

\section{Materials and Methods}

Furosemide raw material (China associated Co. Ltd., China) and HPMC $4000 \mathrm{cp}$ (China associated Co. Ltd., China) were supplied from Ethiopian Pharmaceutical Manufacturing Sh. Co. (EPHARM). Ethylcellulose (Feicheng Rutai, China) was donated by Cadila Pharmaceuticals PLC. Furosemide reference standard (Greenfield pharmaceuticals Co. Ltd., China) was obtained from Food, Medicine and Health care Administration and Control Authority of Ethiopia. Ethanol (Uni. Chem., India), dichloromethane (Research-lab fine Chem. Industries, India), hydrochloric acid (BDH Ltd., England),

*Corresponding author: Mulugeta Fentie, Department of Pharmaceutics and Social Pharmacy, School of Pharmacy, Addis Ababa University, P. O. Box 1176, Addis Ababa, Ethiopia, Tel: 251-111-239-752; E-mail: befentie@gmail.com

Received September 15, 2014; Accepted January 13, 2015; Published January 23, 2015

Citation: Fentie M, Belete A, Mariam TG (2015) Formulation of Sustained Release Floating Microspheres of Furosemide from Ethylcellulose and Hydroxypropyl Methylcellulose Polymer Blends. J Nanomed Nanotechnol 6: 262. doi:10.4172/2157-7439.1000262

Copyright: $\odot 2015$ Fentie M, et al. This is an open-access article distributed under the terms of the Creative Commons Attribution License, which permits unrestricted use, distribution, and reproduction in any medium, provided the original author and source are credited. 
sodium hydroxide (BDH Ltd., England), and Tween 80 (BDH Ltd., England) were all used as received. All chemicals used were analytical grade.

\section{Preparation of microspheres}

Various microsphere formulations were prepared using solvent evaporation method as described by Gattani [8]. A fixed weight $(1 \mathrm{~g})$, but at varied proportions, of ethyl cellulose and HPMC was dissolved in $16 \mathrm{ml}$ of $(1: 1, \mathrm{v} / \mathrm{v})$ dichloromethane and ethanol at room temperature. Weighed amount of furosemide was added to the polymers solution and mixed. The resultant slurry was slowly introduced as a thin stream into a $200 \mathrm{ml}$ of water containing $0.01 \%$ Tween 80 maintained at different temperatures and stirred at different stirring rates using heating magnetic stirrer (Velp Scientifica, Italy) for $1 \mathrm{~h}$ to allow the volatile solvent to evaporate completely. The microspheres formed were filtered, repeatedly washed with distilled water and dried overnight in an oven drier (Kotterman-2711, Germany) at $40^{\circ} \mathrm{C}$.

\section{Characterization of prepared microspheres}

The microspheres were characterized for their particle size, bulk and tapped densities, compressibility index and angle of repose as described below.

\section{Particle size distribution}

The particle size distribution of microspheres was determined using sieving method as described by Yüce and Canefe [9]. Weighed microspheres of each formulation were put in a set of sieves fixed on the universal drive unit (Erweka, AR 402, Germany).

\section{Percentage yield of microspheres}

The production yield of microspheres of each batch was calculated as described by Ghosh [10] using the weight of the final product after drying with respect to the initial total weight of the drug and polymers used for preparation of microspheres, and the percentage production yield was calculated using Equation 2.6.

$$
\text { Yield }(\%)=\frac{\text { Practical mass }(\text { microspheres })}{\text { Theoretical mass }(\text { polymers }+ \text { drug })} \times 100
$$

\section{Drug entrapment efficiency}

Drug entrapment efficiency (DEE) was determined using the method described by Garg and Gupta [11]. Accordingly, a sample of $50 \mathrm{mg}$ drug loaded microspheres of each formulation was taken for evaluation. The weighted microspheres were dissolved in $10 \mathrm{ml}$ dichloromethane in a separating funnel and the drug was repeatedly extracted with aliquots of $0.1 \mathrm{~N} \mathrm{NaOH}$. The extract was transferred to a $100 \mathrm{ml}$ volumetric flask and the volume was made up using $0.1 \mathrm{~N}$ $\mathrm{NaOH}$. The solution was filtered and the absorbance was measured at $271 \mathrm{~nm}$ against $0.1 \mathrm{~N} \mathrm{NaOH}$ as blank. The amount of drug entrapped in the microspheres was calculated by the following formula:

$$
\operatorname{DEE}(\%)=\frac{\text { Amount of drug actually present in the sample }}{\text { Theoretical drug content in the sample }} \times 100
$$

\section{In vitro buoyancy}

In vitro buoyancy studies were carried out for each formulation using a method described by Karthikeyan [12]. $300 \mathrm{mg}$ of drug loaded microspheres were spread over the surface of USP Type II (paddle) dissolution apparatus (Erweka, DT 600, Germany) filled with $900 \mathrm{ml}$ of
$0.1 \mathrm{~N} \mathrm{HCl}$ containing $0.02 \%$ of Tween 80 . The medium was maintained at $37^{\circ} \mathrm{C}$ and agitated with a paddle rotating at $100 \mathrm{rpm}$ for $12 \mathrm{hrs}$. At the end of this period, the layer of buoyant particles on the surface of the medium was collected and the sinking particulates were separated by filtration. Both particle types were dried overnight in an oven drier (Kotterman 2711, Germany) at $40^{\circ} \mathrm{C}$. Dried weights were measured, and buoyancy was determined by the weight ratio of the floating particles to the sum of floating and sinking particles (Equation 2.8).

$$
\text { Buoyancy }(\%)=\frac{\text { Dry weight of floated microspheres }}{\text { Total dry weight of floated and settled microspheres }} \times 100
$$

\section{In vitro drug release study}

A USP type II (paddle) dissolution apparatus (Erweka, DT 600, Germany) was used to study in vitro drug release from microspheres as described elsewhere [13]. Accordingly, an amount of the microspheres equivalent to $10 \mathrm{mg}$ of furosemide filled in a hard gelatin capsule (size 0) was placed in the dissolution medium containing $900 \mathrm{ml}$ of $0.1 \mathrm{~N} \mathrm{HCl}$ and $0.02 \%$ of Tween 80 maintained at $37 \pm 0.5^{\circ} \mathrm{C}$ with paddle rotating at $100 \mathrm{rpm}$. Samples of $10 \mathrm{ml}$ were withdrawn at 0.5 , $1,2,4,6,8,10$ and $12 \mathrm{hrs}$ and filtered. Equal volume $(10 \mathrm{ml})$ of the dissolution medium was replaced in the vessel after each withdrawal to maintain sink condition. Each of the sample solutions were analyzed spectrophotometerically for the drug content at $274 \mathrm{~nm}$. From this, the percentage of drug release was calculated and plotted as a function of time to study the pattern of drug release.

\section{Drug-exciepients interaction study}

FT-IR spectra for pure furosemide and furosemide loaded microspheres formualtion were acquired at room temperature using FTIR spectrophotometer (FTIR-8400S, Shimadzu, Japan) in transmittance mode. The samples were ground in a mortar, mixed with Nujol and placed between two plates of $\mathrm{KBr}$ and compressed to form a thin film. The sandwiched plates were placed in the infrared spectrometer and the spectra were obtained. Scanning was performed between wave numbers $3600-1200 \mathrm{~cm}^{-1}$.

\section{Results and Discussions}

Floating microspheres of furosemide were prepared by the solvent evaporation method from polymers ethyl cellulose and HPMC under varying conditions of drug loading, EC/HPMC ratio, temperature and stirring rate. The prepared floating microspheres were evaluated for different physicochemical tests such as particle size, in vitro buoyancy, drug entrapment efficiency, yield and in vitro drug release behaviors. Preliminary experiments conducted on formulation of the microspheres showed that EC/HPMC of greater than 1:1, temperature of $20-30^{\circ} \mathrm{C}$, and stirring rate of $500-1200 \mathrm{rpm}$ should be used in order to obtain floating microspheres of adequate characteristics.

\section{Particle size analysis}

The particle size of floating microspheres of all the formulations ranged from 718 to $1092 \mu \mathrm{m}$ (Table 1). It was observed that, on increasing the temperature from 20 to $30^{\circ} \mathrm{C}$, the particle size of the microspheres significantly increased $(\mathrm{p}=0.0003)$ from 924 to $976 \mu \mathrm{m}$. This could be related to the higher rate of solvent evaporation upon increased temperature [14]. An increase in drug loading from 300 to $1000 \mathrm{mg}$ also caused significant increase $(\mathrm{p}<0.0001)$ in the average particle size of microspheres from 944 to $1092 \mu \mathrm{m}$. This may be due to diminished shearing efficiency at higher concentration of the drug (higher viscosity) [15]. Increasing EC/HPMC ratio from 1:1 to 9:1 
Citation: Fentie M, Belete A, Mariam TG (2015) Formulation of Sustained Release Floating Microspheres of Furosemide from Ethylcellulose and Hydroxypropyl Methylcellulose Polymer Blends. J Nanomed Nanotechnol 6: 262. doi:10.4172/2157-7439.1000262

Page 3 of 5

\begin{tabular}{|c|c|c|c|c|c|c|c|c|c|}
\hline Code & $\begin{array}{l}\text { Drug amount } \\
\text { (mg) }\end{array}$ & $\begin{array}{c}\text { EC/HPMC } \\
(w / w)\end{array}$ & Temperature $\left({ }^{\circ} \mathrm{C}\right)$ & Stirring rate (rpm) & $\begin{array}{c}\text { Particle size } \\
(\mu \mathrm{m})\end{array}$ & Yield (\%) & DEE (\%) & Buoyancy (\%) & $\begin{array}{l}12 \mathrm{~h} \text { Cum. } \\
\text { release (\%) }\end{array}$ \\
\hline $\mathrm{F} 1$ & 300 & $4: 1$ & 25 & 500 & $944 \pm 7.0$ & $88.2 \pm 3.0$ & $98.3 \pm 0.9$ & $54.1 \pm 4.2$ & $99.8 \pm 5.2$ \\
\hline $\mathrm{F} 2$ & 700 & $4: 1$ & 25 & 500 & $1017 \pm 16.8$ & $89.3 \pm 2.1$ & $96.5 \pm 0.5$ & $64.0 \pm 3.5$ & $62.9 \pm 0.4$ \\
\hline F3 & 1000 & $4: 1$ & 25 & 500 & $1092 \pm 14.0$ & $86.2 \pm 3.3$ & $93.9 \pm 0.8$ & $69.1 \pm 1.9$ & $56.2 \pm 1.2$ \\
\hline $\mathrm{F} 4$ & 300 & $1: 1$ & 25 & 500 & $718 \pm 2.6$ & $34.0 \pm 2.9$ & $86.2 \pm 2.4$ & $8.1 \pm 1.4$ & $99.9 \pm 3.9$ \\
\hline F5 & 300 & $4: 1$ & 25 & 500 & $944 \pm 7.0$ & $88.2 \pm 3.0$ & $98.3 \pm 0.9$ & $54.1 \pm 4.2$ & $99.8 \pm 5.2$ \\
\hline F6 & 300 & $7: 1$ & 25 & 500 & $1070 \pm 6.7$ & $89.4 \pm 3.6$ & $96.0 \pm 1.3$ & $61.0 \pm 2.3$ & $47.9 \pm 2.2$ \\
\hline $\mathrm{F} 7$ & 300 & $9: 1$ & 25 & 500 & $1090 \pm 11.5$ & $91.1 \pm 2.2$ & $98.4 \pm 1.0$ & $77.8 \pm 1.9$ & $41.3 \pm 0.5$ \\
\hline F8 & 300 & $4: 1$ & 20 & 500 & $924 \pm 6.1$ & $95.4 \pm 2.9$ & $98.4 \pm 1.0$ & $50.0 \pm 3.8$ & $100.9 \pm 0.8$ \\
\hline F9 & 300 & $4: 1$ & 25 & 500 & $944 \pm 7.0$ & $88.2 \pm 3.0$ & $98.3 \pm 0.9$ & $54.1 \pm 4.2$ & $99.8 \pm 5.2$ \\
\hline F10 & 300 & $4: 1$ & 30 & 500 & $976 \pm 8.1$ & $63.1 \pm 3.8$ & $86.2 \pm 0.6$ & $46.1 \pm 1.4$ & $95.6 \pm 2.9$ \\
\hline F11 & 300 & $4: 1$ & 25 & 500 & $944 \pm 7.0$ & $88.2 \pm 3.0$ & $98.3 \pm 0.9$ & $54.1 \pm 4.2$ & $99.8 \pm 5.2$ \\
\hline F12 & 300 & $4: 1$ & 25 & 900 & $791 \pm 15.5$ & $93.3 \pm 1.3$ & $97.2 \pm 1.1$ & $52.2 \pm 2.5$ & $100.9 \pm 1.9$ \\
\hline F13 & 300 & $4: 1$ & 25 & 1200 & $721 \pm 9.5$ & $91.5 \pm 4.2$ & $93.2 \pm 1.9$ & $49.3 \pm 1.5$ & $99.8 \pm 3.4$ \\
\hline
\end{tabular}

Table 1: Evaluation of physicochemical characterstics of microspheres prepared at various levels of process and formulation variables.

caused a significant increment $(\mathrm{p}<0.0001)$ in the average particle size of the microspheres that ranged from 718 to $1090 \mu \mathrm{m}$. The size of microspheres was also significantly decreased with increasing agitation. This is because increasing rate of stirring produces higher energy that decreases the droplet sizes, thus producing smaller microspheres [16].

\section{Drug entrapment efficiency}

The drug entrapment efficiency of all the formulations was in the range of 86.2 to $98.4 \%$, indicating high entrapment efficiency for all of the formulations (Table 1). The results showed the entrapment efficiency decreased significantly $(\mathrm{p}<0.0001)$ from 98.4 to $86.2 \%$ as the temperature was increased from 20 to $30^{\circ} \mathrm{C}$. Increased immiscibility between the droplets and the aqueous medium at lower temperatures may contribute to the increased entrapment efficiency at lower temperatures [17]. Entrapment efficiency was also decreased upon increasing furosemide composition. Increasing EC/HPMC ratio from 1:1 to 9:1 showed increased entrapment efficiency of microspheres from 86.2 to $98.4 \%$ ( $\mathrm{p}<0.0001)$. This may be attributed to the rapid hardening of the droplets following increased ethyl cellulose proportion that results in reduced drug diffusion into the aqueous phase [9]. The lower entrapment efficiency of the drug (86.2\%) at lower EC/HPMC ratio (1:1) may be due to the higher amount of HPMC that causes diffusion of the drug into the aqueous phase through enhancing its apparent solubility [18]. Increasing stirring rate also caused a significant decrease $(\mathrm{p}=0.01)$ of entrapment efficiency.

\section{Yield}

The yield for all the formulations of F1 to F13 was determined. Except for formulation F4 with a yield value of $34.1 \%$, the yield of all the other formulations was good being in the range of 63.1 to $95.4 \%$ (Table 1). Yield of microspheres was decreased significantly $(\mathrm{p}<0.0001)$ from 98.4 to $86.2 \%$ as the temperature was increased from 20 to $30^{\circ} \mathrm{C}$. Upon increasing the EC/HPMC proportion from 1:1 to 9:1, a very high significant increase in yield from 34 to $91.1 \%$ ( $\mathrm{p}<0.0001)$ was also observed. The lower yield value (34\%) at lower EC/HPMC (1:1) could be attributed to the possible migration of the hydrophilic polymer (HPMC) into the aqueous phase [19].

\section{In vitro buoyancy}

Average buoyancy in percentage of all the formulations at the end of $12 \mathrm{~h}$ was found to range from 8.1 to $77.8 \%$. Poor percentage of $8.1 \%$ was exhibited with formulation F4 that contains higher proportion of HPMC (EC/HPMC (1:1)). This is likely due to the water permeable nature of HPMC and its tendency of increasing wettability that causes increased amount of liquid medium absorbed replacing the air inside the floating microspheres, thus rendering them less buoyant. In general with increase in the proportion of ethyl cellulose and drug amount, there was a significant increase in the buoyancy percentage. Buoyancy percentage increased from 54.1 to $69.1 \%(\mathrm{p}=0.004)$ upon increasing furosemide composition from 300 to $1000 \mathrm{mg}$, and from 8.1 to $77.8 \%$ $(\mathrm{p}<0.0001)$ as the EC/HPMC proportion was increased from 1:1 to 9:1. The increase in buoyancy upon increasing ethyl cellulose proportion or drug amount could be due to the poor solubility of the polymer and the drug in acidic medium. However, varying conditions of temperature and stirring rate did not show significant changes in percentage buoyancy of microspheres. The results confirmed that high percentage buoyancy could be achieved at higher proportion of ethyl cellulose and drug amount.

\section{In vitro drug release study}

In vitro drug release studies on all the 13 formulations of furosemide floating microspheres were carried out using a USP dissolution apparatus Type II in $0.1 \mathrm{~N} \mathrm{HCl}$ as dissolution medium. The cumulative percent drug release after $12 \mathrm{~h}$ was found to be $99.8,62.9$ and $56.2 \%$ for the formulations F1, F2 and F3 respectively, whereas cumulative percent drug release after $12 \mathrm{~h}$ was $99.9,99.8,47.9$ and 41.3 for formulations F4, F5, F6 and F7, respectively (Table 1). This results show that the cumulative drug release was significantly decreased with increase in drug amount and with increase in ethyl cellulose proportion. The significant decline $(\mathrm{p}<0.0001)$ in cumulative percent of drug release from $99.8 \%$ to $56.2 \%$ as the loading of the drug was increased from $300 \mathrm{mg}$ to $1000 \mathrm{mg}$ could be attributed to the increased composition of the poorly soluble drug and the formation of larger microspheres with increased drug amount. An increase in ethyl cellulose proportion from 1:1-9:1 retards the release rate of the drug, with a very significant cumulative percent drug release decline of 99.85 to $41 \%(\mathrm{p}<0.0001)$ in $12 \mathrm{~h}$. The reason for this retarded drug release may be due to the increased proportion of the hydrophobic polymer ethyl cellulose that increases the polymer matrix density and thus result in increased diffusional path length, leading to a decrease in drug release from the microspheres [17,20,21]. Another factor might be that the smaller microspheres formed at low ethyl cellulose concentration had larger surface area exposed to the dissolution medium, thus, giving rise to faster drug release [17]. The release profiles of Figures 1 and 2 also showed that the difference in release pattern among formulations prepared at varied composition of the drug or polymers ratio was remarkable. Microspheres prepared at varied conditions of temperature and stirring rate were also evaluated for the release pattern. Results 
Citation: Fentie M, Belete A, Mariam TG (2015) Formulation of Sustained Release Floating Microspheres of Furosemide from Ethylcellulose and Hydroxypropyl Methylcellulose Polymer Blends. J Nanomed Nanotechnol 6: 262. doi:10.4172/2157-7439.1000262

Page 4 of 5

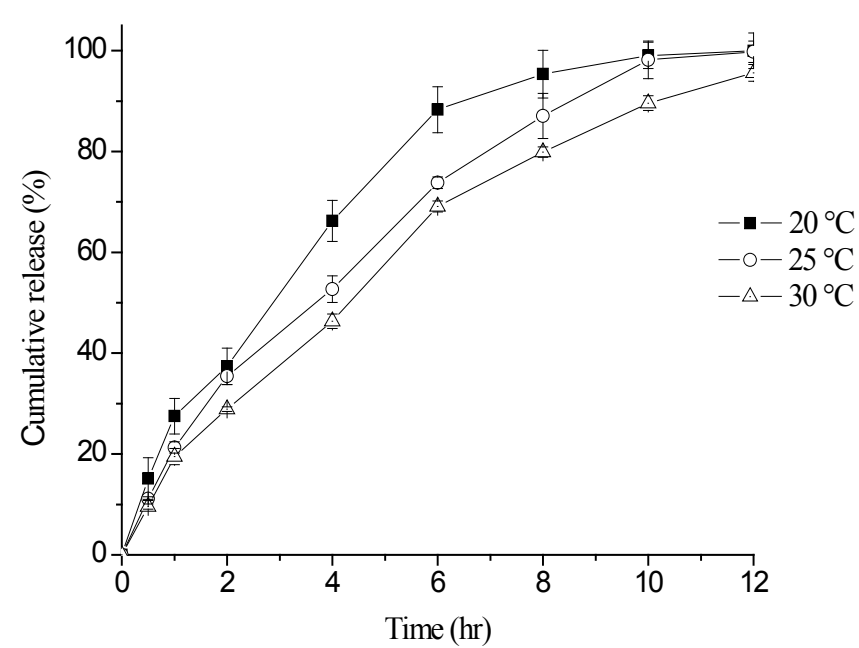

Figure 1: Effect of temperature on the in vitro furosemide release from floating microspheres.

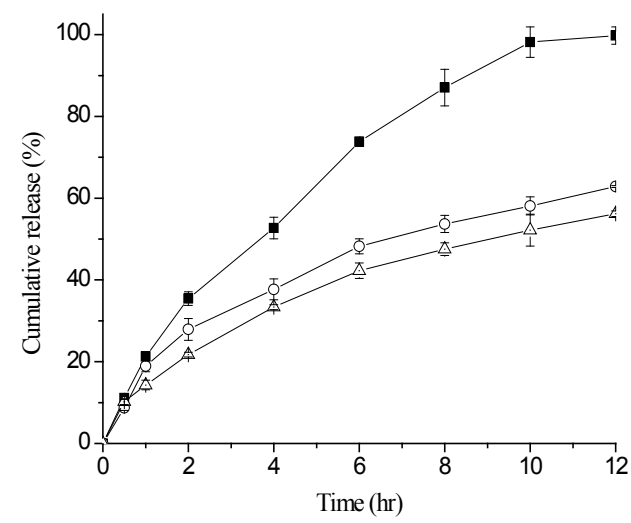

Figure 2: Effect of drug loading on the in vitro furosemide release from floating microspheres.

indicated that changes in temperature and stirring rate did not show significant effects on drug release behavior of furosemide loaded EC/ HPMC floating microspheres (Figures 3 and 4).

\section{Drug-exciepients interaction study}

Drug-excipients interaction was studied using Fourier transformed infrared (FT-IR) spectroscopy. The characteristic peaks of the drug (Figure 5) were observed at wave numbers $3400 \mathrm{~cm}^{-1}, 3350 \mathrm{~cm}^{-1}, 3280$ $\mathrm{cm}^{-1}, 1670 \mathrm{~cm}^{-1}$ and $1560 \mathrm{~cm}^{-1}$ in the functional group region of the pure drug spectrum $[22,23]$. These characterstic peaks in the spectrum correspond to, $3400 \mathrm{~cm}^{-1}$ for $\mathrm{N}-\mathrm{H}$ streching vibration of $\mathrm{Ar}-\mathrm{NHCH}_{2}$ secondary amine, $3350 \mathrm{~cm}^{-1}$ and $3280 \mathrm{~cm}^{-1}$ for $\mathrm{N}-\mathrm{H}$ streching vibrations of Ar- $\mathrm{SO}_{2} \mathrm{NH}_{2}$ primary amines, $1670 \mathrm{~cm}^{-1}$ for carboxilic acid streching vibration of $\mathrm{Ar}-\mathrm{COOH}$ and $1560 \mathrm{~cm}^{-1}$ for $-\mathrm{NH}_{2}$ bending vibration of the $\mathrm{Ar}-\mathrm{SO}_{2} \mathrm{NH}_{2}$ of furosemide [24]. These characteristic peaks also appear in the spectrum of the furosemide microspheres formulation at the same wave numbers indicating that there was no interaction between the drug and formulation excipients [25].

\section{Conclusions}

Floating microspheres of furosemide were successfully prepared by solvent evaporation method. The drug entrapment efficiency of all the formulations was high, in the range of 86.2 to $98.4 \%$. The yield of microspheres production was good, particularly at higher levels of EC/ HPMC ratio and lower temperatures. Drug loading and EC/HPMC ratio showed highly significant effects $(\mathrm{p}<0.0001)$ on drug release and buoyancy of microspheres. The study confirmed various furosemide

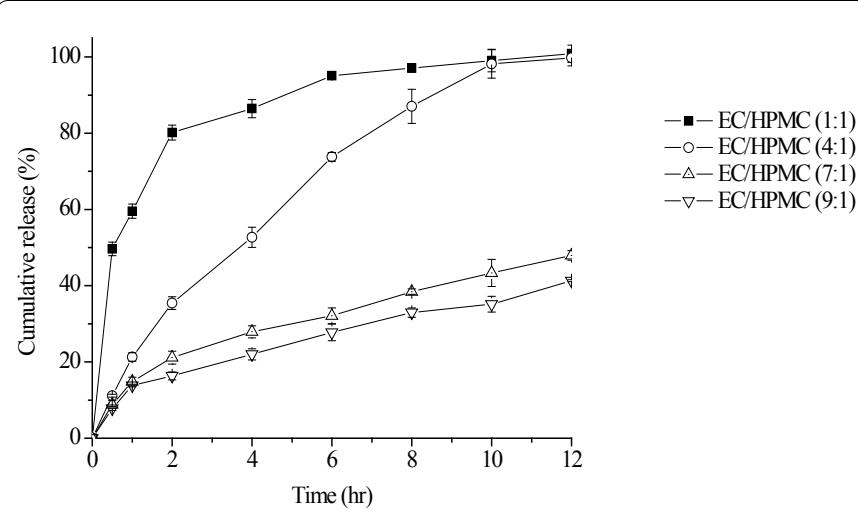

Figure 3: Effect of EC/HPMC ratio on the in vitro furosemide release from floating microspheres.

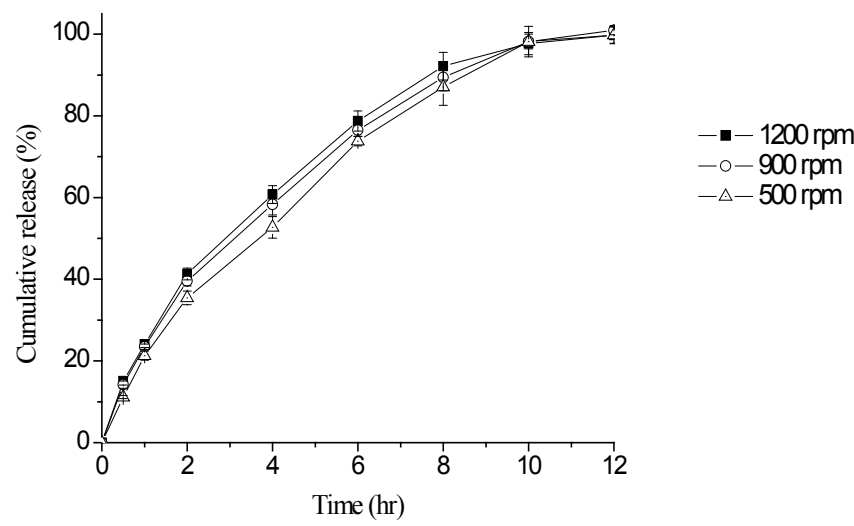

Figure 4: Effect of stirring rate on the in vitro furosemide release from floating microspheres.

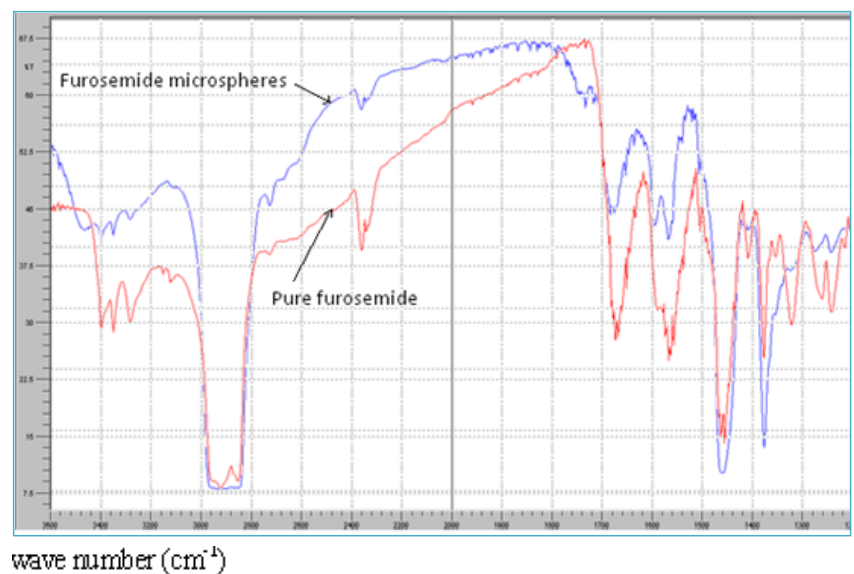

Figure 5: FT-IR spectra of pure furosemide and optimized furosemide loaded microspheres formulation. 
Citation: Fentie M, Belete A, Mariam TG (2015) Formulation of Sustained Release Floating Microspheres of Furosemide from Ethylcellulose and Hydroxypropyl Methylcellulose Polymer Blends. J Nanomed Nanotechnol 6: 262. doi:10.4172/2157-7439.1000262

loaded EC/HPMC microspheres formulations could be developed that effectively sustain the drug release for a desired period by varing the ratio of EC and HPMC, and drug amount. Among the formulations examined $\mathrm{F} 1$ was found to be the best controlled release floating formulation for $12 \mathrm{~h}$. Further, potential of the floating microspheres of furosemide formulations to improve furosemide bioavailability in humans need to be investigated.

\section{References}

1. Gupta S, Udit U, Omray L, Reetesh Y, Soni V (2010) Preparation and characterization of floating drug delivery system of acyclovir. Int J Appl Pharma 2. $7-10$

2. Santini J, Rechards A, Sheidt R, Cima M, Langer R (2000) Microchips as controlled released drug delivery devices. Chem Int Ed 39: 2396-2407.

3. Khan K, Bajpai M (2010) Floating Drug Delivery System: An Overview. Int J PharmTech Res 2: 2497-2505.

4. Granero G, Longhi M, Mora M, Junginger H, Midha K, et al. (2010) Biowaiver monographs for immediate release solid oral dosage forms: Furosemide. J Pharm Sci 99: 2544-2556.

5. KlausnerEA, LavyE, StepenskyD, CserepesE, Barta M, etal (2003)Furosemide pharmacokinetics and pharmacodynamics following gastroretentive dosage form administration to healthy volunteers. J Clin Pharmacol 43: 711-720.

6. Jackson E (2006) Drugs acting Renal and Cardiovascular functions. In; Brunton L, Lazo J, Parker K (eds), Goodman and Gilman's the pharmacological basis of therapeutics. (11thedn), McGraw Hill, New York, USA.

7. Mojtahedzadeh M, Ardakani M, Salehifar E, Najafi A, Khajavi M, et al. (2005) The relationship between pharmacokinetic variables and pharmacodynamic profiles of bolus versus continuous infusion of furosemide in critically ill patients. Daru 13: 127-132.

8. Gattani Y, Kawtikwar P, Sakarkar D (2009) Formulation and evaluation of gastro retentive multiparticulate drug delivery system of aceclofenac. Int $J$ Chem Tech Res 1: 1-10.

9. Yüce M, Canefe K (2008) Indomethacin-loaded microspheres: preparation, characterization and in-vitro evaluation regarding ethylcellulose matrix material. Turk J Pharm Sci 5: 129-142.

10. Ghosh A, Nayak K, Rout P, Nag T, Roy P (2008) Preparation, evaluation and in vitroin vivocorrelation study of lamivudine loaded microspheres. Res J Pharm Tech 1: 353-56.
11. Garg R, Gupta G (2010) Gastroretentive floating microspheres of silymarin Preparation and in vitroevaluation. Trop J Pharma Res 9: 59-66.

12. Karthikeyan D, Karthikeyan M, Ramasamy C (2010) Development of floating microspheres to improve oral bioavalibity of cefpodoxime proxetil. Acta Pharma Sci 52: 101-104.

13. Basu S, Adhiyaman R (2008) Preparation and characterization of nitrendipine loaded eudragit RL100 microspheres prepared by an emulsion-solvent evaporation method. Trop J Pharmaceut Res 7: 1033-1041.

14. Yang Y, Chung T, Bai X, Chan W (2000) Effect of preparation conditions on morphology and release profiles of biodegradable polymeric microspheres containing protein fabricated by double emulsion method. Chem Eng Sci 55 2223-2236.

15. Atyabi F, Mohammadi A, Dinarvand R (2005) Preparation of nimodipine loaded microspheres: Evaluation of parameters. Iran J Pharm Sci 1: 143-152.

16. Basu S, Kavitha K, Rupeshkumar M (2010) Evaluation of ketorolac tromethamine microspheres by chitosan/gelatin complex coacervation. J Pharm Pharm Sci 78: 79-92.

17. Freiberg S, Zhu X (2004) Polymer microspheres for controlled drug release. Int J Pharm 282: 1-18.

18. Nighute A, Bhise S (2009) Preparation and evaluation of rifabutin laded polymeric microspheres. Res J Pharm Tech 2: 371-374.

19. Guyot M, Fawaz F (1998) Nifedipine loaded-polymeric microspheres: preparation and physical characteristics. Int J Pharm 197: 61-74.

20. Das M, Maurya D (2008) Evaluation of diltiazem hydrochloride-loaded mucoadhesive microspheres prepared by emulsification internal gelation technique. Acta Pol Pharm-Drug Res 65: 249-259.

21. Nath $B$, Nath L, Mazumder B, Kumar $P$ (2010) Preparation and characterization of salbutamol sulphate loaded ethyl cellulose microspheres using water-in-oiloil emulsion technique. Iran J Pharm Res 9: 97-105.

22. Bhise S, Chaulang G, Patel P, patel B, Bhosale A, et al. (2009) Superdisintegrants as solubilizing agent. Res J Pharm Tech 2: 387-391.

23. Akinlade B, Elkordy A, Essa E, Elhagar S (2010) Liquisolid systems to improve the dissolution of furosemide. Sci Pharm 78: 325-344.

24. Socrates G (2004) Infrared and Raman characterstic group frequencies: tables and charts. $3^{\text {rd }}$ edtn, John Wiley \& Sons, USA.

25. Karkhile V, Karmarkar R, Sontakke M, Badgujar S, Nemade L (2010) Formulation and evaluation of floating tablets of furosemide. Int J Pharm Res Dev 1: 32-43. 